\title{
¿Preparados para las fake news? Un estudio exploratorio de la comunidad universitaria del Tecnológico de Antioquia
}

\author{
Are you ready for fake news? An exploratory study of the university community \\ Tecnológico de Antioquia
}

Recibido: 17-07-2019 • Aprobado: 03-08-2020 • Página inicial: 175 - Página final: 204

Semillero de investigación en Geopolítica*

Facultad de Ciencias Administrativas y Económicas

Tecnológico de Antioquia

\begin{abstract}
Resumen: este artículo estudia las tendencias de uso de medios de comunicación y redes sociales en el Tecnológico de Antioquia - Institución Universitaria. Concretamente, se examinan los hábitos de consulta, contraste y verificación de noticias de cara a explorar su posible relación con la expansión de las fake news (noticias falsas). Tras aplicar una encuesta estratificada en profesores, administrativos y estudiantes se procede a realizar un análisis estadístico de carácter descriptivo $\mathrm{y}$, posteriormente, un análisis inferencial que involucra la aplicación de la prueba Chi2 y la Prueba de Cramer's V. Los resultados reflejan que los hábitos de consumo, contraste y verificación de noticias no se diferencian por el tipo de vinculación académica y que existen indicios que señalan niveles medio-bajos en hábitos de información, contrastación y envío de información, lo que puede constituir un contexto propicio para la expansión de las fake news.
\end{abstract}

Palabras clave: comportamiento informacional; impacto de la comunicación; fake news; hábitos de información; tecnología de la información y comunicación.
Abstract: This article studies the trends in the use of communication media and social networks at Tecnológico de Antioquia University Institution. Specifically, the habits of consultation, contrast, and verification of news are examined to explore their possible relationship with the expansion of fake news (false news). After applying a stratified survey among teachers, administrators, and students; a descriptive statistical analysis is carried out and, later, an inferential analysis that involves the application of the Chi2 test and the Cramer's Test V. The results reflect that the habits of consumption, contrast, and verification of news are not differentiated by the type of academic connection and that there are clues that indicate medium-low levels of information habits, contrast and sending of information, which can constitute a context conducive to the spread of fake news.

Keywords: Project management; public projects; indicators; public policy.

JEL: 129

\footnotetext{
* Este artículo hace parte de la producción académica del Semillero de Investigación en Geopolítica, cuyos autores se referencian en página contigua.
} 
Pineda, H., Jima-González, A., Paradela-López, M., Acevedo, H., Agudelo, A., Areiza, S., García, C., Giraldo, S., Holguín, C., Loaiza, K., Montoya, A., Ortega, J., Ospina, T. y Parra, J.

\section{Você está pronto para notícias falsas? Um estudo exploratório da comunidade universitária do Tecnológico de Antioquia}

Resumo: este artigo estuda as tendências de uso dos meios de comunicação e redes sociais no Tecnológico de Antioquia - Instituição Universitária. Especificamente, são examinados os hábitos de consulta, contraste e verificação de notícias, a fim de explorar sua possível relação com a expansão de notícias falsas (notícias falsas). Após a aplicação de um inquérito estratificado em professores, gestores e alunos, procede-se a uma análise estatística descritiva e, posteriormente, a uma análise inferencial que envolve a aplicação do teste Chi2 e do Teste V. de Cramer. Os resultados reflectem que os hábitos de Consumo, contraste e apuração de notícias não se diferenciam pelo tipo de vínculo acadêmico e que há indícios que apontam níveis médio-baixo nos hábitos de informação, contraste e envio de informações, o que pode constituir um contexto favorável para a expansão do falso notícia.

Palavras-chave: comportamento informativo; impacto da comunicação; notícias falsas; hábitos de informação; tecnologia da informação e comunicação.

Hernán Pineda Gómez. Docente investigador del Tecnológico de Antioquia, Colombia. hernan.pineda@tdea.edu.co ORCID: https://orcid.org/0000-0002-5691-7390

Alexandra Jima-González. Docente investigadora de la Universidad Yachay Tech, Ecuador. ajima@yachaytech.edu.ec ORCID: https://orcid.org/0000-0002-9719-3069

Miguel Paradela-López. Docente investigador del Tecnológico de Antioquia, Colombia. miguel.paradela@tdea.edu.co ORCID: https://orcid.org/0000-0003-1849-5526

Estudiantes del Tecnológico de Antioquia, Colombia e integrantes del Semillero en Geopolítica:

Henry de Jesús Acevedo Marín, estudiante de Administración Comercial.

haceved1@correo.tdea.edu.co ORCID: https://orcid.org/0000-0002-1750-6070

Ana Isabel Agudelo Velásquez, estudiante de la Licenciatura en Educación Preescolar. anita.agu.velas@gmail.com ORCID: https://orcid.org/0000-0001-9037-4249

Sara Liliana Areiza Villa, estudiante de Negocios Internacionales.

Sara.areiza.10@hotmail.com ORCID: https://orcid.org/0000-0001-5879-9693

César Augusto García Marín, estudiante de Administración Financiera. cesaragm2010@gmail.com ORCID: https://orcid.org/0000-0002-4197-0287

Santiago Andrés Giraldo Valencia, estudiante de Contaduría Pública. santigv.328@gmail.com ORCID: https://orcid.org/0000-0002-2042-9256

Cristian Alonso Holguín Arenas, estudiante de Contaduría Pública.

alonsoholguinarenas1996@gmail.com ORCID: https://orcid.org/0000-0003-1882-8255

Katherin Vanessa Loaiza Seguro, estudiante de Negocios Internacionales.

kathy200789@gmail.com ORCID: https://orcid.org/0000-0001-6865-6805

Antonio José Montoya Acevedo, estudiante de Administración Comercial. anjo7montoya@gmail.com ORCID: https://orcid.org/0000-0002-4932-4191

Juan Pablo Ortega Lyons, estudiante de Negocios Internacionales.

juan.ortega23@correo.tdea.edu.co ORCID: https://orcid.org/0000-0002-7278-9130

Tatiana Ospina Marulanda, estudiante de Contaduría Pública. tati9611@gmail.com ORCID: https://orcid.org/0000-0003-2488-793X

Juan Esteban Parra Tavera, estudiante de Negocios Internacionales.

juanestebanparratavera@gmail.com ORCID: https://orcid.org/0000-0003-1528-9145 


\section{Introducción}

Los cambios tecnológicos de las últimas décadas han revolucionado la comunicación, configurando una serie de nuevas dinámicas y prácticas sociales centradas en el uso masivo de internet. Como resultado, actualmente es posible el acceso a una información rápida, instantánea y completa: con una simple conexión a internet, una persona puede acceder actualmente a las principales fuentes de noticias de todo el mundo. En este sentido, la llegada de las redes sociales ha posibilitado la difusión de manera instantánea de sucesos de la esfera política, económica y social a buena parte de la población mundial.

A su vez, los usuarios de estas nuevas tecnologías han experimentado cambios en su comportamiento informacional, modificando las formas y hábitos de recabar información, así como la forma en la que comunican sus propias vivencias $\mathrm{u}$ opiniones. Por tanto, el surgimiento y penetración de internet ha generado una ruptura con las formas clásicas de comunicación (televisión, prensa escrita, radio, etc.), dando lugar a un nuevo paradigma comunicativo, tanto en la forma de informarse, como en el tipo de interacción con los medios de comunicación o en los mecanismos de difusión de la información. En este contexto, las redes sociales se han configurado como un nuevo paradigma en el proceso de comunicación, ganando una creciente relevancia como un instrumento de relacionamiento y como mecanismo de información (Bernete, 2010).

Más concretamente, la primera encuesta nacional realizada por el Ministerio de Tecnologías de la Información y las Comunicaciones de Colombia (TIC) (2017) reveló que el 64\% de la población colombiana tiene acceso a internet. De estas personas, el $97 \%$ usa internet para comunicarse, empleando, en orden de la relevancia, las redes sociales Facebook (88\%), WhatsApp (87\%), Youtube (48\%), Instagram (34\%) y Twitter (20\%); el 90\% de ellos las utilizan diariamente. Adicionalmente, esta encuesta revela la creciente importancia de las redes sociales como herramienta educativa, puesto que el $44 \%$ de los encuestados reconocieron tal tipo de uso (TIC, 2017).

Por otro lado, esta misma encuesta mostró que el 68\% de las empresas colombianas usan internet como herramienta comercial, llegando hasta el 67\%, las que crearon y sostuvieron redes sociales de la empresa. Más concretamente, las principales redes utilizadas por las empresas fueron Facebook (94\%), Instagram (34\%) y Twitter (27\%). Estos datos evidencian que la intensidad de la penetración de las redes sociales no se ha limitado al ámbito individual y social, sino que también han tenido un fuerte impacto en las empresas y en todos los sectores económicos. 
Al mismo tiempo, este cambio en la forma y medios de informarse implica un gran reto y responsabilidad para la sociedad contemporánea: la instantaneidad de la información, la falta de un control previo y efectivo y la multiplicidad de emisores de información -la mayoría de ellos no periodísticos. Estos desafíos se han cristalizado en una serie de problemáticas, entre las que se destacan la difusión de las fake news, noticias falsas creadas con fines políticos, económicos y humorísticos, enviadas a través de las redes sociales y compartidas por sus lectores -quienes las consideran erróneamente verídicas. Por tanto, existen actualmente problemáticas a la hora de controlar las noticias falsas, lo que ha generado que estas sean utilizadas con fines electorales, arruinando campañas electorales de candidatos o deteriorando la imagen pública de políticos y gestores (Lazer et al., 2018).

Los problemas derivados de esta nueva realidad, y especialmente su impacto en espacios de participación ciudadana como el plebiscito para refrendar el acuerdo de paz en el año 2016 en Colombia (Jima-González et al., 2018; Las noticias falsas que circularon durante la jornada electoral, 2018), han dado lugar a la presente investigación. Más concretamente, los miembros del Semillero en Geopolítica buscaron analizar los hábitos de consulta, verificación y difusión de la información en la comunidad universitaria del Tecnológico de Antioquia - Institución Universitaria. Para ello, se lleva a cabo la implementación de un estudio cuantitativo consistente en la realización de una encuesta estratificada -estudiantes, empleados y docentes- y su posterior análisis descriptivo y correlativo.

El presente artículo se encuentra estructurado en cinco apartados. En el primero se desarrolla el posicionamiento teórico, analizando el impacto de las redes sociales y los hábitos informacionales en la comunidad universitaria. Más concretamente, se plantea la hipótesis desarrollada por el artículo acerca de la forma en la que los hábitos de consumo de información inciden en la propagación de fake news. El segundo apartado presenta el estado de la cuestión, centrado en el análisis de la relación entre las redes sociales y las fake news, así como el comportamiento informacional, tanto en las redes sociales como, específicamente, con las fake news. El tercer apartado corresponde al desarrollo de la metodología, describiendo las particularidades de la encuesta realizada, así como las herramientas utilizadas en el análisis posterior de los datos obtenidos. En cuarto lugar, se desarrolla un apartado concerniente a los resultados obtenidos, exponiendo las evidencias obtenidas a partir de las respuestas a las encuestas, así como las principales correlaciones entres estas. Por último, el quinto apartado expone las conclusiones de la investigación, mostrando la 
influencia de los hábitos de consumo de información en la expansión de las fake news y las futuras líneas de investigación de futuros proyectos.

\section{Marco teórico}

A nivel teórico, el texto reseña el surgimiento y evolución de las redes sociales, su influencia en la sociedad actual y los cambios en el comportamiento informacional que estas han generado en la sociedad. Posteriormente, se explora su posible relación con la aparición de las fake news y el impacto que pueden tener en las dinámicas informativas de la población a través de las redes sociales.

\section{Redes sociales: origen y relevancia en la sociedad}

Las redes sociales y su uso en Internet parten de una teoría intuitiva denominada Seis Grados de Separación, expuesta por el escritor Frigyes Karinthy en 1929 (Ros-Martín, 2009). De acuerdo con esta teoría, el número de conocidos de una persona crece exponencialmente, siguiendo un número de enlaces de una cadena, separados por seis personas. Entre los varios intentos de demostrar científicamente la teoría, se encuentran los estudios realizados por Milgram o Watts -Universidad de Harvard y Universidad de Columbia, respectivamente (como se citó en Ros-Martín, 2009).

Ahora bien, las redes sociales - como se conocen en la actualidad- tienen su origen en la década de los noventa. Gonzálvez (2014) indica que, en el año de 1995, Randy Conrads creó el sitio web llamado classmates.com con el fin de recuperar o mantener algún tipo de comunicación con antiguos compañeros de colegio o universidad. Este portal permitía generar un acercamiento virtual entre una red de contactos que en algún momento hubiesen compartido un lugar físico. Paulatinamente, las plataformas sociales se modernizaron para responder a las necesidades de sus usuarios. Por ejemplo, el sitio web llamado sixdegrees. com permitía a los usuarios no solo crear su perfil y tener una lista de "amigos", sino que también podían compartir mensajes a través de esa plataforma virtual (Pérez, Poveda y López, 2014).

Velásquez (2013) argumenta que la evolución y disolución de las redes sociales tomó lugar, principalmente, en ambientes universitarios, ya que las poblaciones estudiantiles buscan ser parte de diversos fenómenos 'del momento'. Un claro ejemplo de esta dinámica es la creación en 2004 de una de las principales redes sociales, Facebook (Marcos, 2009). Esta plataforma fue diseñada para el uso exclusivo de alumnos de la Universidad de Harvard 
y aunque en un inicio los usuarios de la red social tenían que estar vinculados a la universidad, finalmente Facebook se extendió hacia otras universidades y empresas. En la actualidad, cualquier persona que disponga de un correo electrónico puede unirse a esta red social, que supera actualmente los dos mil millones de usuarios en todo el mundo.

Cabe destacar el elemento generacional en la entrada de las redes sociales, ya que este medio de comunicación es el más utilizado por las generaciones más jóvenes. Las redes sociales con más frecuencia de uso son: WhatsApp 27\%, Facebook 25\%, YouTube 16\%, Twitter 11\%, Instagram 9\%, Google+ 7\%, Tumblr 3\%, Skype 2\% (Cortés, Zapata, Menéndez y Canto, 2015). En cuanto al uso que los usuarios hacen de las redes sociales, destacan los siguientes motivos: crear y mantener amistades, entretenimiento y gestión interna de organizaciones empresariales (Caldevilla, 2010).

No obstante, la incidencia de las redes sociales no se limita a las relaciones personales, puesto que también son utilizadas de manera creciente por las empresas. En un sentido más corporativo, Hütt (2012) afirma que las posibilidades de interacción de las empresas con sus públicos derivadas del uso de redes sociales son muy amplias, sirviendo tanto para contar con un canal de retroalimentación, como para desarrollar un espacio de promoción, información y mercadeo. Adicionalmente, las redes sociales permiten establecer dinámicas menos invasivas y más focalizadas hacia las necesidades y expectativas de los consumidores.

Por tanto, puede afirmarse que toda dimensión de la vida ha experimentado, en menor o mayor grado, una transformación generada por la innovación tecnológica y el uso de las redes sociales (Cornejo y Lourdes, 2011). En este sentido, Pantoja (2011) argumenta que estas redes proporcionan nuevas posibilidades para compensar los grandes vacíos entre las necesidades de comunicación social y los medios necesarios para que dicha comunicación ocurra, priorizando la deslocalización, la imprevisión y la inmediatez.

A pesar del amplio uso que se hace de las redes sociales hoy en día, algunos investigadores advierten sobre las consecuencias negativas de su uso excesivo. Por ejemplo, existe una preocupación creciente por la posible 'despersonalización' de los individuos, así como por un deseo extremo de publicar todos los aspectos de sus vidas en sus redes sociales. Del mismo modo, otros problemas derivados de un uso irresponsable pueden ser la privación del sueño, el descuido de otras actividades importantes y el aislamiento social 
generalizado (Echeburúa y De Corral, 2010). Aunque existen estas críticas, la mayor parte de investigaciones concluyen que las redes sociales y la tecnología no son malas en sí, sino que sus efectos dependen del uso que cada individuo haga de las mismas.

\section{Comportamiento informacional: cambios a partir de las TIC}

Con la entrada de las nuevas tecnologías de la información y las comunicaciones (TIC) y la irrupción del Internet, se presentó un cambio en el comportamiento informacional, entendido como "(...) la conducta, actitud y aptitud de los individuos frente al acceso, uso y comunicación de la información, condicionado por la motivación, el entorno y el contexto del individuo" (Fernández, 2018, p.4). Este cambio es especialmente notorio en las generaciones de personas que nacieron cuando Internet emergió y se empezó a expandir rápidamente -los llamados Millennials (1981-1993) y Generación Z (1994-2005).

En tal sentido, Ávila (2013) sostiene que las TIC han tenido un papel decisivo en el cambio del dinamismo social, cultural y económico. Estas herramientas se han sumergido en las actividades económicas y los usos sociales, situándose comúnmente tanto en el eje de la interacción y el desarrollo entre tecnología y sociedad del conocimiento, como en la simplificación de las tareas del diario común, incluyendo la comunicación interpersonal. En todas estas actividades, las TIC emergen como el nexo que une el mundo online y el mundo off-line (Echeburúa y De Corral, 2010; Ávila, 2013). Configurándose una relación entre lo social y lo tecnológico (2001):

deberíamos pensar sobre los espacios y posibilidades de las relaciones hibridas entre espacios "on-line" y "off-line", asi como el modo en que estas relaciones y espacios se reconfiguran mutuamente". Nuestra huella digital posiblemente nos convierte en más "públicos y abiertos" que sin la presencia de la tecnología. De hecho, en ese continuum virtualpresencial, en el que las TIC mediatizan y modulan nuestros actos comunicativos y nuestras relaciones sociales, haciéndonos más móviles (estamos, y somos, en distintos espacios y lugares a un tiempo), éstas deben poder ser pensadas como legítimas tecnologías de relación. Ya que el rasgo distintivo de las TIC no es la información que circula por ellas sino las relaciones que posibilitan; las TIC son un espacio de relación que vehicula los procesos de comunicación que -gracias a, o mediante ellas- podemos tener con los otros (Como se citó en Gil-Juárez, VallLlovera \& Feliu, 2010). 
Consecuentemente, las TIC no solo mediatizan la comunicación, sino también las relaciones, los afectos, las experiencias, el consumo y la expresión cultural. Por ende, la tecnología puede llegar a modificar el modo de pensar y sentir de los individuos, incidiendo así en el desarrollo de nuevas identidades (Gil-Juárez et al., 2010). De esta forma, el comportamiento informacional de los individuos cambia radicalmente, ya que las TIC dan lugar a la creación de espacios en los que la interacción es parte del proceso en el que se adquiere nueva información, gracias a que la presencialidad y la virtualidad fluyen de manera continua.

Debido a todos estos cambios, el desarrollo de las TIC llega a fracturar las estructuras clásicas de la comunicación e información, dando lugar a un nuevo paradigma en donde el público tiene la oportunidad de ser parte de la noticia, comentar e influir en su posible propagación (Yilin, 2014). Esto último -y en vista de la creciente falta de credibilidad de los medios tradicionales de información- ha hecho que el comportamiento informacional de las generaciones más jóvenes se incline por una comunicación más horizontal, como la que ofrecen las plataformas de internet: directa y sin necesidad de intermediarios. Un ejemplo claro de este proceso es la 'desacralización' de los diarios impresos como fuente primaria de información en el contexto digital, que se evidencia en un decreciente número de sus lectores y la preferencia por la información obtenida a través de las redes sociales (Gil-Juárez et al., 2010).

Otra cuestión que toma relevancia a la luz de la llegada de las TIC en el comportamiento informacional de los jóvenes es la facilidad y rapidez a la hora de informarse. Esto último, unido a la idea de que las noticias deben ser gratuitas -concebidas no como un producto, sino como un servicio públicopodría iniciar una desvalorización de la información (Casero-Ripollés, 2012), sobre todo tomando en cuenta que cada vez hay más portales web que proveen este servicio -aunque puedan hacerlo con una calidad cuestionable.

En este sentido, las generaciones actuales ya no se enfrentan a una situación de "información escasa", sino que, por el contrario, la principal problemática actual es la de distinguir la información confiable y veraz en un contexto de sobreabundancia e hipervelocidad en las comunicaciones. Por tal motivo, cobra relevancia educar a los individuos en cuanto a su consumo mediático para lograr evitar problemas como la sobresaturación de información, la sobrecarga cognitiva y la hiperconexión a las tecnologías. De esta forma, las personas podrían llegar a consumir apropiadamente la información: filtrándola y categorizándola según su relevancia y confiabilidad (Serrano-Puche, 2013). 


\section{Fake news y hábitos informacionales}

El término fake news (noticias falsas) se define como “(...) informaciones publicadas deliberadamente en medios digitales que no han sido comprobadas ni verificadas, que carecen de fuentes identificadas y que no cuentan con la supervisión de un editor" (Pauner, 2018, p.300). Actualmente, las fake news han logrado un alto grado de notoriedad debido a su utilización en campañas políticas en Brasil y Estados Unidos, pero su uso y estudio es tan antiguo como la prensa misma. Así lo indica Waisbord (2018), quien resalta que:

(...) las mentiras y la distorsión en la comunicación interpersonal son tan antiguas como la retórica; la propaganda de larga escala es tan vieja como la persuasión moderna de las masas. Lo que es diferente es la velocidad, escala y proliferación masiva al igual que el acceso o consumo de información falsa diseminada en plataformas digitales como Facebook o Twitter (p.2).

El uso de las fake news -al menos a nivel conceptual- no es un fenómeno reciente y su popularidad tiene que ver más con los procesos de masificación de la información desarrollados con la llegada del internet (Fernández, 2017). La relación entre las fake news e internet ha cobrado gran relevancia en los últimos años, a partir de cambios en los comportamientos informacionales y su incidencia en agendas mediáticas (Gobbi, 2017). Parte de este cambio radica en que las personas prefieren a las plataformas digitales como primer canal de búsqueda a la hora de informarse (Yilin, 2014). En este sentido, Richter (2018) argumenta que esto ocurre porque internet y las redes sociales posibilitan que cualquier persona produzca contenido y sea posible la interacción instantánea, configurándose como una especie de micrófono con el que antes no se contaba y brindando una ilusión de poder en el manejo y consumo de la información.

De manera complementaria, Caro (2015) plantea que este elemento -que cualquier persona sea capaz de crear contenido nuevo y difundirlo de manera efectiva- se configura como uno de los retos actuales en el uso del internet. Más concretamente, la alta posibilidad de acceso y la poca capacidad que se tiene para distinguir la información falsa de la verdadera por parte de quienes consumen los contenidos, genera un contexto que propicia la difusión de noticias falsas o de muy baja calidad.

Por otro lado, un elemento a destacar en este contexto de auge de plataformas digitales -y a la vez clave en la difusión de noticias falsas- tiene que ver con 
el tipo de contenido que circula. Fernández (2017) destaca que, en los portales actuales, lo visual (fotos, vídeos, gifs) prima sobre lo textual, lo que implica la difusión de un contenido más cercano a las emociones:

Esta transición de un esquema de aprehensión de la realidad basada en la dedicación de tiempo, en el esfuerzo, en la lectura reposada propia del documento impreso se desmantela dando paso a una nueva forma en los medios digitales. Este modelo consiste en una lectura sintética, donde predomina la visualización de videos, de preferencia por mensajes cortos, de contenidos con escasa carga informativa (Fernández, 2017, p.7).

La propagación de noticias falsas se enmarca entonces en un contexto de masificación de la información y de cambios en los hábitos de obtención de información. Por tal motivo, las fake news se han convertido en un fenómeno con capacidad de incidir en dinámicas sociales, políticas e incluso económicas, constituyéndose en uno de los principales desafíos para las democracias contemporáneas.

\section{Estado de la cuestión}

A continuación, se reseña un grupo de investigaciones que giran en torno al objeto de estudio abordado en la investigación. La revisión de la literatura busca explicar la influencia de las fake news en el uso que los individuos hacen de las redes sociales, así como en la forma en que las utilizan a la hora de informarse de los eventos cotidianos. Por tanto, estas investigaciones buscan analizar la relevancia de la comprensión de los hábitos informacionales y, además, de los mecanismos de incidencia y procesos de propagación de las fake news.

Comenzando con Fernández (2017), esta autora investigó las formas en las que las personas acceden y comparten información en la era digital y el rol que las plataformas digitales tienen en su propagación. En este sentido, resalta cómo las noticias falsas no son un fenómeno nuevo, sino que la novedad únicamente radica en la forma en que estas se reproducen en las redes sociales. Una característica relevante es que en estas plataformas se diluye la fuente, ya que quien recibe la noticia asume un rol pasivo frente a su contrastación, sobre todo cuando el contenido es de índole emocional. Por tanto, este tipo de contenido puede manipularse para impactar en los asuntos políticos y las campañas electorales, donde suele hacerse una apelación directa a cuestiones emocionales fácilmente transmisibles a través de noticias falsas. 
En esta misma línea, Reis \& Oliveira (2017) resaltan que la veracidad de la información que circula actualmente en los medios digitales se ha convertido en un problema que afecta no sólo a las personas, sino también a las empresas. Para llegar a esta afirmación, los investigadores analizan el funcionamiento de las redes sociales, específicamente centrados en el ritmo en que se produce y circula la información por estos medios y en el alcance que logran tener. Como resultado de este análisis, destacan la rapidez con la que se puede compartir información inexacta, distorsionada o falsa en cuestión de minutos, logrando llegar rápidamente a miles de personas.

Por su parte, Rojas, Fernández, Rodríguez y Guillén (2018) exponen las problemáticas de las fake news en los entornos educativos y proponen como solución la creación de un software con la capacidad de diferenciar las noticias verdaderas de las falsas. Para ello, los investigadores parten de un proyecto propuesto por el gobierno italiano, que consiste en formar a los alumnos para que sean capaces de identificar fake news. Su investigación se apoya en el estudio realizado por Vosoghi, Roy \& Aral (2018), en donde se demuestra de manera fehaciente que las noticias falsas se difunden hasta veinte veces más rápido en las redes sociales, y que los responsables de ello son las propias personas y no los bots. También se demuestra que los ámbitos donde con mayor intensidad impactan las noticias falsas son los de la política, el terrorismo, los desastres naturales, la información financiera y la ciencia.

Valero y Oliveira (2018) sostienen que una solución posible para la propagación de fake news es la creación de algoritmos específicos, la verificación por profesionales o la colaboración de muchas personas para poder confrontarlas. Además, muestran que la intención de engañar al público es un problema global, reforzado por el uso del internet y por el hecho de que las personas no cuenten con formación específica destinada a reconocer la información falsa. En las conclusiones de su estudio, los autores plantean que el conocimiento y la educación son las mejores armas para combatir las fake news, que compaginen la instalación de mecanismos de control informático con una aproximación crítica hacia la información recibida.

Siguiendo esta línea, Lotero, Romero y Pérez (2018) realizaron una investigación en torno al fact-checking como herramienta para combatir la desinformación y la propagación de las fake news en Colombia. A través del estudio de la plataforma para combatir noticias falsas llamada 'colombiacheck. com', estos autores afirman que las fake news no sólo influyen en el ámbito político, sino que pueden afectar al ciudadano de manera directa. La conclusión 
principal de la investigación es que los medios de comunicación enfrentan un gran reto en la era digital, dado que no sólo les corresponde el papel de actualización de plataformas, sino que también deben diseñar estrategias para evitar la desinformación y generar programas que garanticen la alfabetización digital de los usuarios.

Finalmente, es importante destacar que la propagación de noticas falsas ha cobrado una importante relevancia en contextos electorales. En esta vía, Muñoz (2017) realizó una investigación en la que caracterizó y analizó catorce noticias que surgieron en un periodo de cuatro meses durante la campaña presidencial en Estados Unidos en 2016. Como metodología para el análisis diseñó un modelo de noticia seria y de calidad con varios elementos principales de análisis que son: la precisión y la calidad de la información, las fuentes, la fecha y la autoría.

Como resultado, se encontró que las catorce noticias seleccionadas no hacían uso correcto de la información y contaban con muy pocos elementos periodísticos. Además, las noticias contaban con elementos estilísticos que simulan el estilo de una noticia verdadera. Para concluir, la autora observa que durante el periodo de estudio se generaron una serie de fake news que contribuyeron a la desinformación, privilegiando el beneficio de un candidato en particular en detrimento de sus adversarios políticos.

Los elementos expuestos en este apartado muestran la importancia creciente que tienen las noticias falsas en la sociedad contemporánea. Del mismo modo, ilustran la importancia de continuar investigando sobre hábitos de información, verificación y sobre cómo se comparte la información, especialmente en entornos educativos que posibiliten trazar pautas para lograr avanzar en la construcción de estrategias que permitan discernir la información que se encuentra en las redes sociales.

\section{Metodología}

El presente estudio se desarrolló en la Institución Universitaria Tecnológico de Antioquia - entidad del orden departamental, con más de 38 años de experiencia en el campo educativo en el Área Metropolitana del Valle del Aburra y el departamento de Antioquia. Actualmente cuenta con 34 programas en los niveles Técnico Profesional, Tecnológico, Profesional Universitario y Posgrado.

Los programas en el Valle de Aburra se ofertan en la sede central de Robledo, así como de forma descentralizada, en los municipios de Itagüí y Copacabana. 
Con respecto al perfil del estudiante, este varía en gran medida por el tipo de jornada que desarrolla (diurna o mixta). En la primera jornada, la población característica, en su mayoría, son jóvenes entre los 18 y 25 años. Por el contrario, en la jornada mixta asisten, en gran medida, estudiantes de mayor edad que cumplen una jornada laboral y posteriormente asisten a clases en horarios compatibles. No obstante, las evidencias de las encuestas no muestran ninguna significación específica con respecto a los resultados sobre las fake news.

El tipo de estudio realizado es de carácter transversal, ya que el instrumento de medición se aplicó una única vez en la muestra escogida. La muestra poblacional, calculada con un $95 \%$ de grado de confianza, fue obtenida a partir de la población del Tecnológico de Antioquia - Sede Robledo, categorizándola de la siguiente manera:

Tabla 1.

Población y muestra utilizada en la aplicación de encuestas

\begin{tabular}{lcc}
\hline & Estudiantes & Profesores y Personal Administrativo \\
\hline Población & 8361 & 1094 \\
\hline Muestra & 368 & 269 \\
\hline
\end{tabular}

Elaboración propia con base en la información suministrada por la Oficina de Talento Humano del Tecnológico de Antioquia (2019).

En este sentido, se implementó una encuesta compuesta por trece preguntas que se aplicó a un total de 637 personas del plantel educativo: docentes, personal administrativo y estudiantes (347 mujeres y 290 hombres). Del mismo modo, el promedio de edad de los estudiantes que respondieron es de 22 años, mientras que el de los docentes es de 38 años.

Centrados en la metodología del análisis, este se llevó a cabo en un doble estudio descriptivo e inferencial. En primer lugar, se procedió a la aplicación de técnicas de estadística descriptiva, con el fin de obtener las características generales de la población y poder conocer las especificidades de cada grupo con respecto al uso de medios de comunicación y hábitos de consulta de noticias. En segundo lugar, se llevó a cabo el análisis de la información utilizando estadística inferencial, con el propósito de hallar correlaciones estadísticas entre las diferentes variables. De esta forma, se pudo estudiar con un mayor grado de profundidad la relación que existe entre el uso de medios de comunicación, el hábito de consulta de noticias y la posible divulgación de noticias falsas. 


\section{Contexto}

Pineda, H., Jima-González, A., Paradela-López, M., Acevedo, H., Agudelo, A., Areiza, S., García, C., Giraldo, S., Holguín, C., Loaiza, K., Montoya, A., Ortega, J., Ospina, T. y Parra, J.

Las dos fases de análisis se apoyaron en el programa estadístico Data Analysis and Statistical Software (Stata). Específicamente, se aplicaron diferentes pruebas estadísticas para comprobar la relación entre las diferentes variables: la prueba Chi 2, con el propósito de verificar la dependencia entre las variables estudiadas; y la Prueba Cramer's V, para comprobar la fuerza y dirección de la correlación entre las variables.

\section{Análisis de resultados}

Tal y como se expuso en el apartado metodológico, la presente sección consta de dos fases de análisis. Por un lado, los datos de las encuestas fueron analizados con el fin de estudiar las tendencias de la muestra con relación al uso de medios de comunicación, hábitos de consulta y de compartir noticias. Por otro lado, se aplicaron técnicas de estadística inferencial para explorar la relación entre el uso de medios de comunicación, el hábito de consulta de noticias y la posible divulgación de noticias falsas.

\section{Análisis descriptivo}

\section{Uso de medios de comunicación y redes sociales}

Uno de los primeros datos relevantes obtenidos del análisis de la encuesta tiene que ver con el alto porcentaje de los encuestados que tienen redes sociales. Como se puede observar en la Figura 1, este porcentaje llega al 96,7\%, es decir que, de un total de 637 encuestados, solamente 21 no tienen redes sociales. Diferenciando entre docentes y alumnos, puede afirmarse que no existen diferencias significativas entre ambos, ya que el 99,18\% de los alumnos tiene redes sociales frente a un $93,31 \%$ de los profesores (Tabla 2 ).

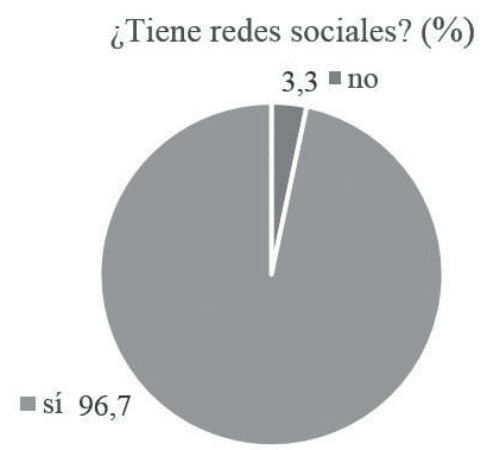

Figura 1. Porcentaje de la muestra que tiene redes sociales.

Elaboración propia con base en los resultados de las encuestas. 
Tabla 2.

Frecuencia y porcentaje que tiene redes sociales (desagregado)

\begin{tabular}{lcccc}
\hline & \multicolumn{2}{c}{ Sí } & \multicolumn{2}{c}{ NO } \\
\hline Alumnos & $\mathrm{f}$ & $\%$ & $\mathrm{f}$ & $\%$ \\
\hline Profesores/Administrativos & 365 & 99,18 & 3 & 0,82 \\
\hline
\end{tabular}

Elaboración propia con base en los resultados de las encuestas.

Un segundo elemento que destacar, tiene que ver con la utilización de los medios de comunicación, tal y como puede observarse en la Tabla 3. Entre estos, las redes sociales son las más utilizadas por la población encuestada: el 22,56\% dedica más de 4 horas al día, marcando una clara diferencia con la radio (3,54\%), la televisión $(2,84 \%)$ y la prensa escrita-digital $(1,76 \%)$. De hecho, puede observarse cómo más del $58 \%$ de la población dedica como mínimo 2 horas diariamente al uso de las redes sociales. De estos datos puede concluirse que actualmente las redes sociales se han convertido en un elemento fundamental en el relacionamiento de la población estudiada y, por tanto, de la información que esta recibe.

Tabla 3.

Tiempo que se dedica a los medios de comunicación (\%)

\begin{tabular}{lccc}
\hline & $2-3 \mathrm{~h}$ & $3-4 \mathrm{~h}$ & más de $4 \mathrm{~h}$ \\
\hline Redes Sociales & 20,62 & 15,58 & 22,56 \\
\hline TV & 15,3 & 5,68 & 2,84 \\
\hline Radio & 6,28 & 4,67 & 3,54 \\
\hline Prensa escrita/digital & 16,48 & 4,48 & 1,76 \\
\hline
\end{tabular}

Elaboración propia con base en los resultados de las encuestas.

Profundizando más en la cuestión, si bien un alto porcentaje de los encuestados utiliza las redes sociales más de cuatro horas al día, la Tabla 4 explora qué redes sociales son aquellas utilizadas con mayor frecuencia. En este sentido, se observa cómo el programa de mensajería instantánea WhatsApp es el medio de comunicación más utilizado, pues un 34,26\% lo utiliza más de cuatro horas al día. Otras redes sociales que son utilizadas con frecuencia son la plataforma de vídeos YouTube y la red de fotos y vídeos Instagram, con un 10,44\% y $10,6 \%$, respectivamente. 
La mayor utilización del WhatsApp, frente a otras aplicaciones, puede tener que ver con la multiplicidad de usos que puede darse a la aplicación: desde la comunicación instantánea o la participación en grupos, hasta el reenvío de cadenas de mensajes informativos. De hecho, la facilidad de comunicarse con grupos o personas puede tener que ver con que esta aplicación haya sido utilizada a menudo en la difusión de fake news.

Tabla 4.

Tiempo que se dedica a las redes sociales (\%)

\begin{tabular}{lccc}
\hline & $2-3 \mathrm{~h}$ & $3-4 \mathrm{~h}$ & más de $4 \mathrm{~h}$ \\
\hline Facebook & 15,79 & 6,28 & 5,6 \\
\hline Twitter & 4,42 & 2,12 & 0,58 \\
\hline Instagram & 17,92 & 10,42 & 10,6 \\
\hline YouTube & 21,25 & 10,44 & 10,44 \\
\hline WhatsApp & 21,86 & 18,43 & 34,26 \\
\hline
\end{tabular}

Elaboración propia con base en los resultados de las encuestas.

Por todo lo anterior, se puede establecer que la muestra encuestada comparte algunas tendencias en cuanto al uso de medios de comunicación y redes sociales. Así, puede afirmarse que la mayor parte de los docentes y alumnos del Tecnológico de Antioquia - IU utilizan redes sociales, recurriendo a ellas en un promedio de más de 2 horas al día, principalmente a través de la aplicación WhatsApp.

Hábitos de consumo y verificación de noticias

Una vez abordado el tiempo que se le dedica a las redes sociales, se analizan los hábitos de lectura y verificación de los encuestados, para lo que se pregunta por la forma en que consumen las noticias que leen y cómo las verifican antes de darles difusión o credibilidad. Tal y como se observa en la Figura 2, un 49,29\% de los encuestados dice leer el texto completo cuando lee una noticia, frente a un $40,82 \%$ que solo ojea el texto y un $9,89 \%$ que únicamente lee el titular. 
Hábitos de consumo de noticias (\%)

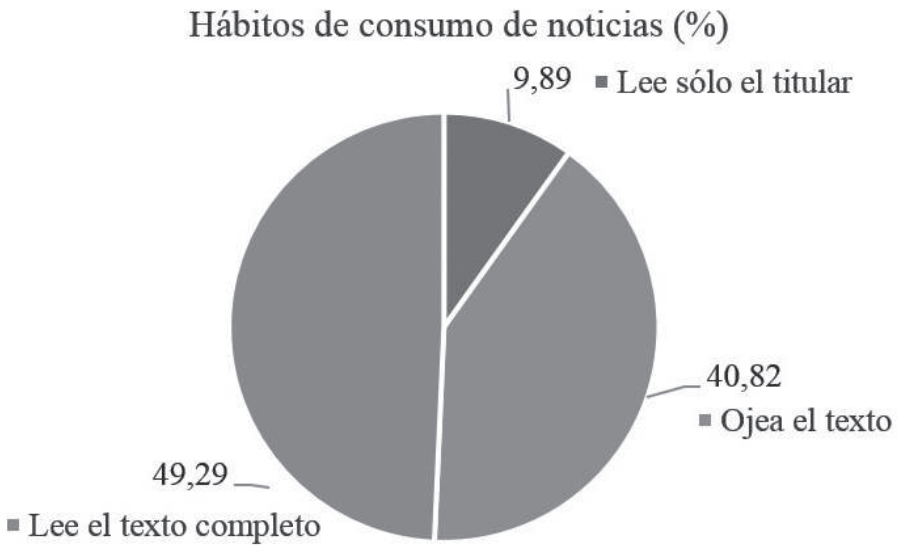

Figura 2. Hábitos de consumo de noticias (\%).

Elaboración propia con base en los resultados de las encuestas.

Intuitivamente, el equipo investigador esperaba encontrar diferencias significativas de estos patrones entre alumnos y profesores/administrativos, derivados del diferente nivel académico, del tipo de actividad laboral y de la edad. Sin embargo, los datos desagregados en la Tabla 5 muestran que el comportamiento entre las dos sub-muestras guarda similitudes importantes: el $50 \%$ de los alumnos dice leer el texto completo frente a un 48,33\% de 10 s profesores/administrativos. Considerando el margen de error del $5 \%$, no puede tomarse esta diferencia de 1,66 puntos porcentuales como estadísticamente representativa. Este dato invita a la reflexión, acerca de la forma en la que las nuevas tecnologías han permeado transversalmente los hábitos de información de la población en su conjunto.

Tabla 5.

Hábitos de consumo de noticias (desagregados)

\begin{tabular}{lcccccc}
\hline & \multicolumn{2}{c}{ Lee sólo el titular } & \multicolumn{2}{c}{ Ojea el texto } & \multicolumn{2}{c}{ Lee el texto completo } \\
\hline & $\mathrm{f}$ & $\%$ & $\mathrm{f}$ & $\%$ & $\mathrm{f}$ & $\%$ \\
\hline Alumnos & 39 & 10,6 & 145 & 39,4 & 184 & 50 \\
\hline $\begin{array}{l}\text { Profesores } \\
\text { /Administrativos }\end{array}$ & 24 & 8,92 & 115 & 42,75 & 130 & 48,33 \\
\hline
\end{tabular}

Elaboración propia con base en los resultados de las encuestas. 


\section{Contexto}

Pineda, H., Jima-González, A., Paradela-López, M., Acevedo, H., Agudelo, A., Areiza, S., García, C., Giraldo, S., Holguín, C., Loaiza, K., Montoya, A., Ortega, J., Ospina, T. y Parra, J.

Con respecto a los hábitos de verificación de noticias, según lo reflejado en la Figura 3, la mayoría de los encuestados $(55,42 \%)$ manifestaron fijarse en la fuente y en la vigencia de las noticias en el momento de leerlas. Por el contrario, un $24,96 \%$ de estos eran conscientes parcialmente de la vigencia $(8,95 \%)$ o de la fuente $(16,01 \%)$; y únicamente un $19,62 \%$ manifestaba no fijarse ni en la fuente ni en la vigencia de las noticias.

Estos datos, por tanto, reflejan que la mayoría de los encuestados tienen una actitud crítica hacia la información que reciben, cuestionando la fuente de la que esta proviene, así como su temporalidad. No obstante, los investigadores se plantean la posible existencia de un sesgo de los encuestados que favorezca una respuesta positiva, tanto por parte de los estudiantes universitarios (que han recibido capacitación en comprensión lectora) como en profesores (de los que se espera una actitud crítica).

Hábitos de verificación de noticias (\%)

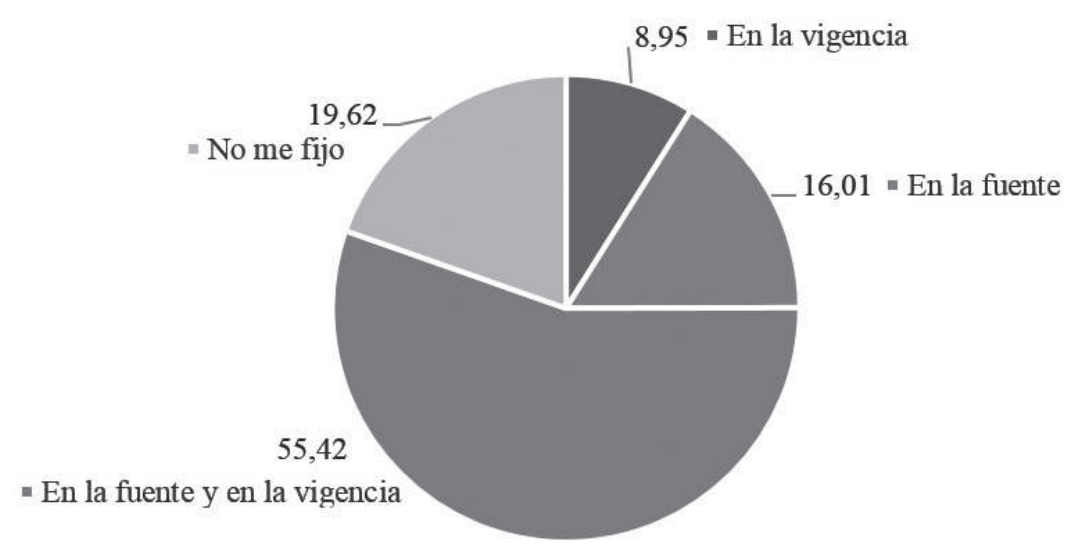

Figura 3. Hábitos de verificación de noticias (\%).

Elaboración propia con base en los resultados de las encuestas.

Al igual que la tendencia anterior, el equipo investigador esperaba encontrar diferencias marcadas entre alumnos y profesores/administrativos. A pesar de que sigue existiendo una tendencia parecida entre los dos colectivos, la Tabla 6 evidencia un comportamiento diferente con respecto a la verificación. Así, mientras un 50,54\% de alumnos revisa la fuente y la vigencia de las noticias que consume, la cifra se eleva hasta un $62,08 \%$ en los profesores/administrativos, suponiendo una diferencia porcentual de aproximadamente 12 puntos. 
Tabla 6

Hábitos de verificación de noticias (desagregados)

\begin{tabular}{lcccccccc}
\hline & Vigencia & \multicolumn{2}{c}{ Fuente } & \multicolumn{2}{c}{ Fuente y vigencia } & \multicolumn{2}{c}{ No me fijo } \\
\hline & $\mathrm{f}$ & $\%$ & $\mathrm{f}$ & $\%$ & $\mathrm{f}$ & $\%$ & $\mathrm{f}$ & $\%$ \\
\hline Alumnos & 42 & 11,41 & 62 & 16,85 & 186 & 50,54 & 78 & 21,2 \\
\hline Profesores/Administrativos & 15 & 5,58 & 40 & 14,87 & 167 & 62,08 & 47 & 17,47 \\
\hline
\end{tabular}

Elaboración propia con base en los resultados de las encuestas.

Siguiendo con el análisis de las tendencias de verificación de la información, la Figura 4 refleja la respuesta acerca de si los encuestados verifican la información que leen en una noticia, consultando otras similares. Frente a esto, el 53,53\% de la población encuestada manifiesta efectivamente contrastarlo, frente al 46,47\% que no lo hace. De nuevo, los investigadores se plantean la posibilidad de la existencia de un sesgo, dado el entorno académico en el que se desenvuelven los encuestados.

Cuando usted lee una noticia, ¿contrasta la información que lee en ella con otras fuentes? (\%)

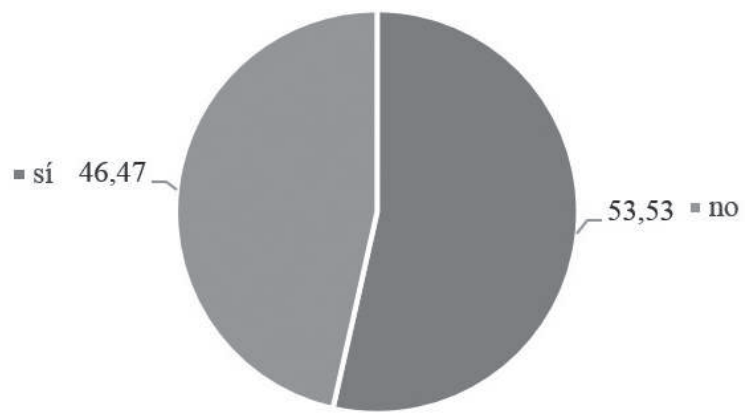

Figura 4. Hábitos de contraste de noticias (\%).

Elaboración propia con base en los resultados de las encuestas.

Analizando los datos de manera desagregada, la Tabla 7 muestra diferencias significativas entre alumnos y docentes/administrativos: mientras que solo el $48,64 \%$ de los estudiantes tienden a contrastar la información, la cifra se eleva hasta el $60,22 \%$ en los docentes y administrativos, alcanzando un diferencial de 12 puntos porcentuales. Tomando esta tabla junto con la anterior, se abre la posibilidad de considerar la existencia de diferentes pautas de verificación entre profesores y alumnos. Esta posibilidad es analizada a través de la prueba Chi 2 en la parte inferencial del artículo. 
Tabla 7

Hábitos de contraste de noticias (desagregado)

\begin{tabular}{lcccc}
\hline & \multicolumn{2}{c}{ Sí } & \multicolumn{2}{c}{ NO } \\
\hline Alumnos & $\mathrm{f}$ & $\%$ & $\mathrm{f}$ & $\%$ \\
\hline Profesores/Administrativos & 179 & 48,64 & 189 & 51,36 \\
\hline
\end{tabular}

Elaboración propia con base en los resultados de las encuestas.

Con base en lo anterior, puede afirmarse que la población general del Tecnológico de Antioquia - IU se caracteriza por leer el texto completo de las noticias, verificar su vigencia y fuente y contrastar la noticia con otras fuentes. No obstante, también cabe señalar la existencia de pautas de verificación diferenciadas entre la población docente-administrativa y el estudiantado.

\section{Hábitos de compartir noticias}

Según los datos expuestos en la Figura 5, se puede ver que un 39,87\% de la población encuestada comparte noticias con sus contactos después de leerlas. De entre ellos, según la Figura 6, un 46,06\% lo hace inmediatamente después de leer la noticia, frente al $31,5 \%$ que las comparte después de haber visto noticias similares, un 18,9\% que lo hace después de unas horas y un 3,54\% que lo hace pasado unos días. Destaca en esta pregunta cómo existe una preeminencia de la inmediatez en la difusión de noticias, lo que sin duda es reflejo de los avances en intercomunicación de las redes sociales.

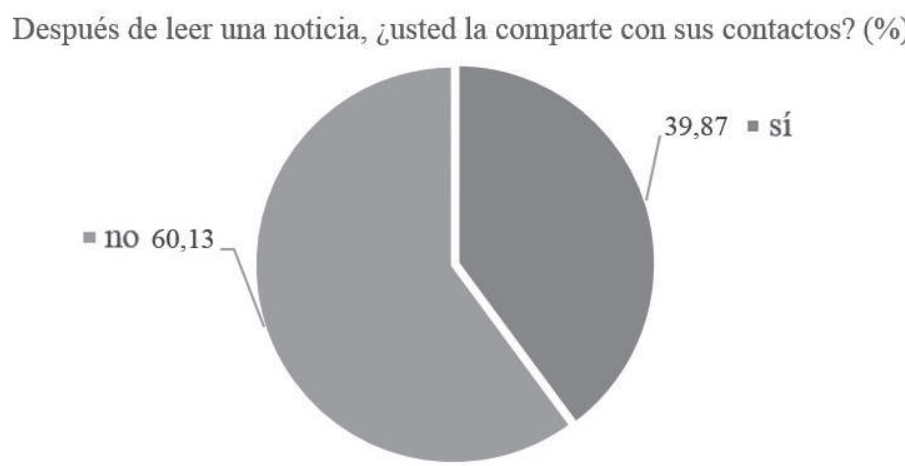

Figura 5. Hábitos de compartir noticias (\%).

Elaboración propia con base en los resultados de las encuestas. 


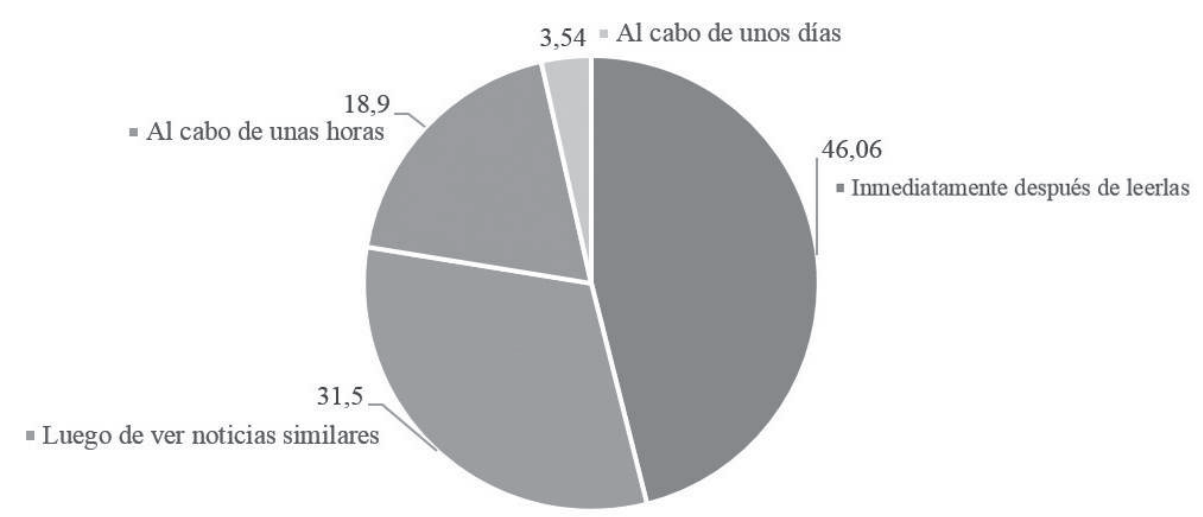

Figura 6. Momento de compartir noticias (\%).

Elaboración propia con base en los resultados de las encuestas.

Por otro lado, un análisis desagregado de los datos, expuestos en la Tabla 8 , refleja una diferencia de aproximadamente cinco puntos entre docentesadministrativos $(38,32 \%)$ y alumnos $(42,01 \%)$. Por otro lado, la Tabla 9 muestra cómo un $48,94 \%$ de alumnos y un $42,48 \%$ de alumnos/administrativos dice compartir inmediatamente las noticias, elevando la diferencia entre ambos grupos hasta seis puntos. De nuevo, la separación entre profesoresadministrativos y alumnos puede ser significativa de la existencia de diferentes tendencias informativas, si bien esta hipótesis debe ser analizada de manera inferencial en el apartado posterior.

Tabla 8 .

Hábitos de compartir noticias (desagregado)

\begin{tabular}{lcccc}
\hline & \multicolumn{2}{c}{ Sí } & \multicolumn{2}{c}{ NO } \\
\hline Alumnos & f & $\%$ & F & $\%$ \\
\hline Profesores/Administrativos & 141 & 38,32 & 227 & 61,68 \\
\hline & 113 & 42,01 & 156 & 57,99 \\
\hline
\end{tabular}

Elaboración propia con base en los resultados de las encuestas. 
Tabla 9.

Momento de compartir noticias (desagregado)

\begin{tabular}{lccccccccc}
\hline & \multicolumn{2}{c}{ Inmediatamente } & \multicolumn{2}{c}{$\begin{array}{c}\text { Luego de } \\
\text { ver noticias } \\
\text { similares }\end{array}$} & \multicolumn{2}{c}{$\begin{array}{c}\text { Al cabo de } \\
\text { unas horas }\end{array}$} & \multicolumn{2}{c}{$\begin{array}{c}\text { Al cabo de } \\
\text { unos días }\end{array}$} \\
\hline & $\mathrm{f}$ & $\%$ & $\mathrm{f}$ & $\%$ & $\mathrm{~F}$ & $\%$ & $\mathrm{f}$ & $\%$ \\
\hline Alumnos & 69 & 48,94 & 37 & 26,24 & 30 & 21,28 & 5 & 3,55 \\
\hline $\begin{array}{l}\text { Profesores } \\
\text { /Administrativos }\end{array}$ & 48 & 42,48 & 43 & 38,05 & 18 & 15,93 & 4 & 3,54 \\
\hline
\end{tabular}

Elaboración propia con base en los resultados de las encuestas.

Después de haber explorado los hábitos de consumo, verificación y formas de compartir noticias, se pueden esbozar dos tendencias generales. En primer lugar, los sujetos encuestados suelen leer el texto completo de las noticias, fijándose en la fuente y en la vigencia de las mismas. En segundo lugar, aquellos encuestados que revelan compartir las noticias que reciben -directa $o$ indirectamente- a través de sus contactos, tienden a hacerlo inmediatamente, después de haber leído la noticia en cuestión. La causalidad y correlación entre estas tendencias son objeto de estudio en el apartado siguiente.

\section{Análisis inferencial}

Como anteriormente se expuso, el análisis descriptivo del artículo ha demostrado la existencia de ciertas diferencias entre docentes-administrativos y estudiantes del Tecnológico de Antioquia - IU. No obstante, dado que la mera causalidad no implica necesariamente la existencia de correlación, el objetivo de este apartado es el de realizar un análisis inferencial de las distintas variables analizadas. Para ello se van a realizar dos tipos de pruebas: la Prueba Pearson Chi 2 -diseñada para medir la independencia de las variables-y la Prueba Cramer's V -empleada con el fin de medir la intensidad de la asociación entre variables nominales, en caso de existir.

Con este propósito, en primer lugar, se procedió a aplicar la Prueba Chi 2 para verificar la existencia o no de independencia entre si el encuestado era alumno, profesor o personal administrativo (variable vinculación) y las variables revisadas en el apartado anterior: 
- Tenencia de redes sociales (redsoc).

- Hábitos de consumo de noticias (medcons).

- Verificación de noticias (fuvig).

- Contraste de noticias (contras).

- Hábitos de compartir noticias (comp).

- Momento de compartir noticias (comphab).

Como se evidencia en la Tabla 10, solamente la variable tenencia de redes sociales y la variable contraste de noticias tienen asociación significativa con la variable vinculación. ${ }^{1}$ Es decir, que el hecho de tener una vinculación determinada no guarda relación con los hábitos de consulta, verificación de noticias, hábitos de compartir noticias y momento de hacerlo. Este resultado descarta, en parte, la hipótesis anteriormente sostenida, de que existían diferentes tendencias entre profesores y alumnos, lo que evidencia que actualmente hay una transversalidad en los hábitos de consumo, verificación y formas de compartir información.

Tabla 10.

Prueba de Chi 2 para establecer asociación entre la vinculación y las variables de interés

\begin{tabular}{lc}
\hline \multicolumn{1}{c}{ Variable } & Pr \\
\hline Redes sociales (redsoc) & 0,000 \\
\hline Hábitos de consumo de noticias (medcons) & 0,620 \\
\hline Verificación de noticias (fuvig) & 0,011 \\
\hline Contraste de noticias (contras) & 0,004 \\
\hline Hábitos de compartir noticias (comp) & 0,347 \\
\hline Momento de compartir noticias (comphab) & 0,231 \\
\hline
\end{tabular}

Elaboración propia con base en los resultados de las encuestas.

En segundo lugar, se procedió a explorar la posible asociación entre otras variables de interés, con el objetivo de medir la existencia y el sentido de las posibles correlaciones. Concretamente, se busca conocer si el hábito de consumo de noticias (medcons) se asocia con el hecho de efectivamente compartirlas o no (comp). A pesar de que la Prueba de Chi 2 indica que sí hay una asociación, esta no es significativa, puesto que el resultado de la prueba Cramer's V es inferior a 0,6 (Tabla 11). Por tanto, no puede afirmarse que el hecho de leer las noticias de

\footnotetext{
${ }^{1}$ Según la Prueba Chi 2, para que se rechace la hipótesis nula $\left(\mathrm{h}_{0}=\right.$ las dos variables son independientes entre sí), el Pr debe ser menor a 0,005.
} 
manera completa influya intensamente en la difusión que se da de ellas, lo que supone que no hay un hábito de informarse correctamente -leyendo la noticia de manera completa- antes de compartir las noticias que se consumen.

Tabla 11.

Chi 2 y Cramer's V para las variables medcons y comp

\begin{tabular}{lcc}
\hline Variables & $\mathrm{Chi}^{2}$ & Cramer's V \\
\hline medcons, comp & 0,000 & 0,1659 \\
\hline
\end{tabular}

Elaboración propia con base en los resultados de las encuestas.

Por último, se analiza un doble análisis de los siguientes dos grupos de variables, para conocer la intensidad de su relación:

- Hábitos de contraste (contras) y hábitos de compartir (comphab).

- Hábitos de verificación (fuvig) y hábitos de contraste (contras).

En este sentido, se procedió a realizar la Prueba Chi 2 entre los dos grupos de variables, evidenciando la existencia de un relacionamiento entre ambas. No obstante, el posterior análisis a través de la Prueba Cramer's V reporta una intensidad entre dicha asociación demasiado baja como para afirmar la existencia de una relevancia significativa. La Tabla 12 resume los datos estadísticos de la relación entre estas variables:

Tabla 12.

Chi 2 y Cramer's V para las variables contras-comphab y fuvig-contras

\begin{tabular}{lcc}
\hline Variables & $\mathrm{Chi}^{2}$ & Cramer's V \\
\hline contras, comphab & 0,000 & 0,2913 \\
\hline fuvig, contras & 0,000 & 0,3200 \\
\hline
\end{tabular}

Elaboración propia con base en los resultados de las encuestas.

Por tanto, no puede afirmarse que el hecho de contrastar las noticias consumidas influya en el momento en el que se comparte a través de las redes sociales. Del mismo modo, tampoco se han reportado evidencias suficientes acerca de la relación entre la atención que prestan a la vigencia y fuente de las noticias, con los hábitos de contraste de las noticias. Al igual que el análisis anterior, 
este revela la falta de un hábito de informarse correctamente, verificando y contrastando la información consumida antes de compartirla con otras personas.

\section{Conclusiones}

El presente artículo ha realizado un análisis de los hábitos de información, consumo y formas de compartir noticias de los docentes, administrativos y estudiantes del Tecnológico de Antioquia - IU. Para ello, se realizaron encuestas estratificadas, revelando unos datos que posteriormente fueron revisados a través del análisis inferencial -concretamente a través de la Prueba Chi 2 y la Prueba Cramer's V. En conjunto, se ha permitido conocer de manera detallada cómo esta población recibe, analiza y comparte la información obtenida, al mismo tiempo que analiza el papel que las redes sociales juegan en este proceso.

Después de haber realizado el análisis descriptivo de los datos recolectados a través de la encuesta, se pudo extraer las características de la muestra con relación al uso de redes sociales, y hábitos de consumo, verificación y formas de compartir noticias. Un primer aspecto a destacar es que la mayoría de los sujetos encuestados tienen redes sociales y las utilizan como principal medio de información, revelando la influencia transversal que las nuevas tecnologías tienen en los hábitos de consumo de la información. De hecho, las redes sociales se han convertido en un elemento fundamental, ya que más del $50 \%$ de los encuestados dedicaban al menos dos horas al día a su uso, especialmente del WhatsApp, que une la información con la comunicación social. En este sentido, puede señalarse una primera problemática en la información de los encuestados: a pesar de que usan las redes sociales para comunicarse o divertirse, constituye indirectamente su principal mecanismo de información.

En segundo lugar, la encuesta ha revelado que, si bien prácticamente la mitad de los profesores y estudiantes leen las noticias que consultan cuidadosamente, una parte importante las ojea o únicamente lee el titular. Del mismo modo, prácticamente este mismo porcentaje revisa la fuente y la vigencia de las noticias, aunque aproximadamente un $45 \%$ no revisa la fuente o la vigencia. Esto implica que, si bien existen ciertas tendencias a analizar de forma crítica la información recibida, una parte relevante de los encuestados (en torno al $40-45 \%$ ) realiza una lectura superficial de la misma sin verificar su fiabilidad.

En tercer lugar, se ha mostrado cómo una parte significativa de la población estudiada comparte habitualmente la información que consulta, de los cuales, prácticamente, la mitad lo hacen de manera inmediata. Esta tendencia 
refleja cómo la llegada de las redes sociales ha generado una nueva pauta de información en los usuarios, en donde prima la inmediatez y la visualidad, ${ }^{2}$ incluso a costa de su fiabilidad.

Por otro lado, el análisis inferencial de los datos revela que no hay una diferencia relevante en las tendencias de consumo, verificación y formas de compartir noticias entre alumnos y profesores-administrativos, lo que confirmaría el impacto transversal que la tecnología y, más concretamente, las redes sociales han tenido sobre los diferentes colectivos. Esto tiene sentido, si se considera que las redes sociales han sido utilizadas, tanto por personas adultas como por jóvenes, prácticamente el mismo tiempo.

Por último, los resultados inferenciales obtenidos entre las variables analizadas, si bien mostraron inicialmente un grado de dependencia, posteriormente evidenciaron una intensidad demasiado baja como para afirmar una influencia directa. Estos resultados señalan la inexistencia de un hábito de verificación y contraste anterior a la divulgación frente a terceras personas, lo que puede significar un nicho favorable a la expansión de fake news entre la población del Tecnológico de Antioquia - IU.

Resulta necesario, en definitiva, una mayor investigación sobre las características de otros colectivos con el fin de trazar líneas de comparabilidad que permitan establecer el impacto de las redes sociales en la sociedad. No obstante, aunque no se hayan podido evidenciar relaciones de influencia directa desde una perspectiva estadística, el trabajo sí ha mostrado cómo existe un grupo representativo de población estudiantil y profesoral en el Tecnológico de Antioquia - IU que, por sus mejorables pautas de información, puede verse expuesta a fake news y, de manera involuntaria, colaborar en su expansión.

En este sentido, el presente artículo evidencia cómo el campo de la información constituye un punto de mejora necesaria para el funcionamiento correcto de la sociedad, especialmente en el ámbito del debate político-democrático. Para ello, resulta imprescindible una mejora sustancial de la actitud crítica hacia la información recibida, desarrollando unos hábitos de cotejo y revisión que sirvan de "cortafuegos" ante cualquier información falsa a la que la población pueda verse expuesta.

2 Las redes sociales que cuentan con más seguimiento son aquellas que facilitan el envío y visualización de contenido visual o auditivo: WhatsApp, Facebook e Instagram. 


\section{Referencias}

Ávila, W. (2013). Hacia una reflexión histórica de las TIC. Revista Hallazgos, $X(19), 213-233$.

Bernete, F. (2010). Usos de las TIC, Relaciones sociales y cambios en la socialización de las y los jóvenes. Revista de Estudios de juventud, 88, 97-114.

Caldevilla, D. (2010). Las Redes Sociales. Tipología, uso y consumo de las redes 2.0 en la sociedad digital actual. Documentación de las Ciencias de la Información, 33, 45-68.

Caro, M. (2015). Información y verdad en el uso de las redes sociales por parte de adolescentes. Teoría de la educación, 27(1), 187-199.

Casero-Ripollés, A. (2012). Más allá de los diarios: el consumo de noticias de los jóvenes en la era digital. Revista Comunicar, 20(39), 151-158.

Cornejo, M. y Lourdes, M. (2011). Redes sociales y relaciones interpersonales en internet. Fundamentos en humanidades, XII(24), 219-229. Recuperado de https://www.redalyc.org/html/184/18426920010/

Cortés, R., Zapata, A., Menéndez, V. y Canto, P. (2015). El estudio de los hábitos de conexión en redes sociales virtuales, por medio de la minería de datos. Innovación Educativa, XV(68), 99-114.

Echeburúa, E. y De Corral, P. (2010). Adicción a las nuevas tecnologías y a las redes sociales en jóvenes: un nuevo reto. Revista Adicciones, XXII(2), 91-95.

Fernández, N. (2017). Fake news una oportunidad para la alfabetización mediática. Revista Nueva Sociedad, 269, 66-77. Recuperado de https:// dialnet.unirioja.es/servlet/articulo? codigo $=6054552$

Fernández, V. (2018). Comportamiento informacional: nuevos escenarios, nuevos desafíos. Páginas $a \& b, 3,3-16$.

Gil-Juárez, A., Vall-Llovera, M. \& Feliu, J. (2010). ICT Consumption and Emergent Subjectivities: New Problems? Psychosocial Intervention, 19(1), 19-26. 
Gobbi, J. (2017). Información digital y nuevas formas de construcción de la agenda de noticias. Sociales en Debate, 12, 29-36.

Gonzálvez, J. (2014). Redes sociales y lo 2.0 y 3.0. Madrid, España: Visión Libros.

Hütt, H. (2012). Las redes sociales: una nueva herramienta de difusión. Revista Reflexiones, XCI(2), 121-128.

Jima-González, A., Paradela-López, M., Pineda-Gómez, H., Areiza-Villa, S. L., García-Marín, C. A., Loaiza-Seguro, K. y Tavera, J. (2018). El Acuerdo de Paz de la Habana. Un estudio de percepción en la población del Tecnológico de Antioquia-IU. Revista En-Contexto, 6(9), 96-120. Recuperado de https://ojs.tdea.edu.co/index.php/encontexto/\%20article/view/507

Las noticias falsas que circularon durante la jornada electoral. (2018). El Tiempo. Recuperado de https://www.eltiempo.com/elecciones-colombia-2018/ jurados-y-votantes/fake-news-difundidas-durante-la-segunda-vueltapresidencial-231116

Lazer, D., Baum, M., Benkler, Y., Berinsky, A., Greenhill, K., Menczer, F. \& Schudson, M. (2018). The science of fake news. Science, 359(6380), 1094-1096.

Lotero, G., Romero, L., y Pérez, M. (2018). 'Fact-checking' vs. 'fake news': periodismo de confirmación como recurso de la competencia mediática contra la desinformación. Revista científica en el ámbito de la Comunicación Aplicada, 8(2), 295-316.

Marcos, M. (2009). Evolución de los servicios de redes sociales en internet. El profesional de la información, XVIII(5), 552-557.

Ministerio de Tecnologías de la Información y las Comunicaciones (TIC). (2017). Primera GRAN Encuesta TIC / 2017, estudio de acceso, uso y retos de las TIC en Colombia. Recuperado de https://colombiatic.mintic. gov.co/679/articles-74002_Presentacion.pdf

Muñoz, P. (2017). Medios de comunicación y posverdad: Análisis de las noticias falsas en elecciones presidenciales de EE. UU. de 2016. (Tesis de Maestría). Universidad Autónoma de Barcelona, España. Recuperado de https://ddd.uab.cat/pub/trerecpro/2017/hdl_2072_293813/TFM_Prisci1la_Munoz.pdf 
Pantoja, A. (2011). Los nuevos medios de comunicación social: las redes sociales. Tejuelo, 12, 218-226.

Pauner, C. (2018). Noticias falsas y libertad de expresión e información. El control de los contenidos informativos en la red. Teoría y Realidad Constitucional, 41, 297-318.

Pérez, M., Poveda, M. y López, M. (2014). El fenómeno de las redes sociales: evolución y perfil del usuario. EduPsykhé - Revista de psicología y educación, XIII(1), 93-118.

Reis, A. \& Oliveira, L. (2017). The current state of fake news: challenges and opportunities. Procedia Computer Science, 121, 817-825. Recuperado de https://www.researchgate.net/publication/321820496_The_current_ state_of_fake_news_challenges_and_opportunities

Richter, U. (2018). El Ciudadano digital: Fake news y posverdad en la era de internet. Bogotá D.C, Colombia: Editorial Océano.

Rojas, D., Fernández, P., Rodríguez, M. y Guillén, A. (2018). Plataforma de entrenamiento para detectar FakeNews en los Recursos Educativos como Internet. Revista de Enseñanza y Aprendizaje de Ingeniería de Computadores, 8, 185-196. Recuperado de https://dialnet.unirioja.es/ servlet/articulo? codigo $=6710903$

Ros-Martín, M. (2009). Evolución de los servicios de Redes Sociales en Internet. El Profesional de la Información, 18(5), 552-558.

Serrano-Puche, J. (2013). Una propuesta de dieta digital: repensando el consumo mediático en la era de la hiperconectividad. Fonseca Journal of Communication, 7, 156-175.

Valero, P. y Oliveira, L. (2018). Fake News: Una revisión sistemática de la literatura. Observatorio Journal, 54-78. doi: https://doi.org/10.15847/ obsOBS12520181374

Velásquez, C. (2013). Análisis de las redes sociales del alumnado de la Universidad de San Carlos de Guatemala (Tesis de doctorado). Universidad de Almería, España. 
Vosoghi, S., Roy, D. \& Aral, S. (2018). The spread of true and false news online. Science, 359, 1146-1151. Recuperado de https://science.sciencemag.org/ content $/ 359 / 6380 / 1146$

Waisbord, S. (2018). Truth is what happens to news. Journalism Studies, 19(13), 1866-1878. doi: 10.1080/1461670X.2018.1492881

Yilin, D. (2014). La transformación del periodismo tradicional en el nuevo entorno digital (Tesis de maestría). Universidad Autónoma de Barcelona, España.

\section{Para citar este artículo:}

Pineda, H., Jima-González, A., Paradela-López, M., Acevedo, H., Agudelo, A., Areiza, S., García, C., Giraldo, S., Holguín, C., Loaiza, K., Montoya, A., Ortega, J., Ospina, T. y Parra, J. (2020). ¿Preparados para las fake news? Un estudio exploratorio de la comunidad universitaria del Tecnológico de Antioquia. EnContexto, 8(12), 175-204. 